\title{
Line-field confocal optical coherence tomography for high-resolution noninvasive imaging of skin tumors
}

\author{
Arnaud Dubois \\ Olivier Levecq \\ Hicham Azimani \\ David Siret \\ Anaïs Barut \\ Mariano Suppa \\ Véronique del Marmol \\ Josep Malvehy \\ Elisa Cinotti \\ Pietro Rubegni \\ Jean-Luc Perrot
}




\title{
Line-field confocal optical coherence tomography for high-resolution noninvasive imaging of skin tumors
}

\author{
Arnaud Dubois,,${ }^{\mathrm{a}, \star}$ Olivier Levecq, ${ }^{\mathrm{b}}$ Hicham Azimani, ${ }^{\mathrm{b}}$ David Siret, ${ }^{\mathrm{b}}$ Anaïs Barut, ${ }^{\mathrm{b}}$ Mariano Suppa, ${ }^{\mathrm{c}}$ \\ Véronique del Marmol, ${ }^{\mathrm{C}}$ Josep Malvehy, ${ }^{\mathrm{d}}$ Elisa Cinotti, ${ }^{\mathrm{e}}$ Pietro Rubegni,, ${ }^{\mathrm{e}}$ and Jean-Luc Perrot ${ }^{f}$ \\ aUniversité Paris-Saclay, Institut d'Optique Graduate School, Laboratoire Charles Fabry, Palaiseau, France \\ ${ }^{\mathrm{D} D A M A E}$ Medical, Paris, France \\ 'Hôpital Erasme, Université Libre de Bruxelles, Department of Dermatology, Brussels, Belgium \\ dUniversity Hospital Clinic of Barcelona, Barcelona, Spain \\ eUniversity of Siena, Department of Medical, Surgical and Neurological Sciences, Dermatology Unit, Siena, Italy \\ fUniversity Hospital of Saint-Etienne, Saint-Etienne, France
}

\begin{abstract}
An optical technique called line-field confocal optical coherence tomography (LC-OCT) is introduced for high-resolution, noninvasive imaging of human skin in vivo. LC-OCT combines the principles of time-domain optical coherence tomography and confocal microscopy with line illumination and detection using a broadband laser and a line-scan camera. LC-OCT measures the echo-time delay and amplitude of light backscattered from cutaneous microstructures through low-coherence interferometry associated with confocal spatial filtering. Multiple A-scans are acquired simultaneously while dynamically adjusting the focus. The resulting crosssectional B-scan image is produced in real time at 10 frame/s. With an isotropic spatial resolution of $\sim 1 \mu \mathrm{m}$, the LC-OCT images reveal a comprehensive structural mapping of skin at the cellular level down to a depth of $\sim 500 \mu \mathrm{m}$. LC-OCT has been applied to the imaging of various skin lesions, in vivo, including carcinomas and melanomas. LC-OCT images are found to strongly correlate with conventional histopathological images. The use of LC-OCT as an adjunct tool in medical practice could significantly improve clinical diagnostic accuracy while reducing the number of biopsies of benign lesions. ๑ The Authors. Published by SPIE under a Creative Commons Attribution 3.0 Unported License. Distribution or reproduction of this work in whole or in part requires full attribution of the original publication, including its DOI. [DOI: 10.1117/1.JBO.23.10.106007]
\end{abstract}

Keywords: optical coherence tomography; skin cancer; optical imaging; confocal microscopy.

Paper 180456R received Jul. 17, 2018; accepted for publication Oct. 1, 2018; published online Oct. 23, 2018.

\section{Introduction}

Skin cancer is the most commonly occurring cancer in humans, with an incidence that has steadily increased worldwide in recent decades. ${ }^{1}$ The prognosis of advanced melanoma is poor, and nonmelanoma skin cancers are associated with high morbidity due to skin invasion. ${ }^{2}$ In addition to causing negative health outcomes, skin cancer poses a significant economic burden. Despite recent therapeutic advances, the factors that have the greatest impact on prognosis remain early detection and complete removal of cancer tissue before the onset of deep invasion and metastasis development. ${ }^{3}$ The standard diagnostic procedure begins with a visual examination of the superficial structures of the skin. ${ }^{4}$ If the lesion is suspicious, a biopsy is performed and the tissue is processed for histological examination. The result of this time-consuming procedure is that nearly $60 \%$ of all skin biopsies result in benign diagnoses. ${ }^{5}$ On the other hand, $20 \%$ of all skin cancers (including roughly a third of melanomas) are missed at an early stage. ${ }^{6}$ Given these issues, improved diagnostic modalities using noninvasive imaging techniques have been developed to provide earlier, more accurate detection of malignant lesions. ${ }^{7-9}$ The clinically available techniques capable of in vivo skin imaging with the highest

*Address all correspondence to: Arnaud Dubois, E-mail: arnaud.dubois@ institutoptique.fr spatial resolution are reflectance confocal microscopy (RCM), optical coherence tomography (OCT), and nonlinear optical microscopy.

RCM is an optical technique that provides en face sectional views of the skin with a spatial resolution comparable with histology of $\sim 1 \mu \mathrm{m} .{ }^{7}$ RCM has been employed for the diagnosis of both melanocytic and nonmelanocytic lesions, where it has been proven to increase the diagnostic accuracy. ${ }^{10-13}$ The main limitation of RCM, however, is a relatively weak penetration of only $\sim 200 \mu \mathrm{m}$ in skin, preventing imaging of structures located in the reticular dermis. ${ }^{7}$ Another major issue is the interpretation of the RCM sections due to their en face orientation, i.e., perpendicular to the conventional vertically oriented histological sections.

OCT is an interferometric optical imaging modality initially introduced in the clinical field of ophthalmology ${ }^{14}$ and used for the first time in dermatology in $1997 .{ }^{15}$ OCT produces crosssectional images of skin with a resolution of a few micrometers, significantly lower than that of RCM. ${ }^{16}$ However, OCT has a higher penetration depth in skin than RCM, on the order of $1 \mathrm{~mm} .^{7}$ The possibility of evaluating OCT images in a vertically oriented view makes them easier to compare with conventional histological sections. OCT has been applied to the diagnosis of nonmelanoma skin cancers. ${ }^{17-19}$ The diagnosis of malignant melanoma using OCT, however, is not as accurate as using $\mathrm{RCM}$, mainly because of the insufficient imaging resolution of OCT. $^{20}$ 
Nonlinear optical microscopy is an imaging modality based on nonlinear interactions of light with biological tissues. ${ }^{21}$ Compared with OCT, nonlinear microscopy offers a better spatial resolution, similar to that of $\mathrm{RCM}^{9}$ Advances in developing nonlinear (multiphoton) excitation microscopes with contrast mechanisms, such as second harmonic generation and stimulated Raman scattering, further allow visualization of skin morphology and function based on molecular-level signatures of biological molecules. Major limitations of this technique are the orientation of the images (en face sections, like RCM), the small field of view (FOV) (smaller than that of $\mathrm{RCM}$ ), and the relatively shallow penetration in skin $(\sim 200 \mu \mathrm{m})$. This technology is significantly more expensive than RCM and OCT, and it is not available as a handheld device.

The current clinically available imaging techniques do not entirely meet the need in dermatology for early, noninvasive detection of skin cancers, particularly melanomas. As a response to this challenge, we have developed an optical technique called line-field confocal (LC) OCT, with the goal of combining the advantages of both RCM and OCT in terms of spatial resolution, penetration, and image orientation. LC-OCT is an echo-based imaging modality that measures the time of flight and amplitude of light backscattered from the sample microstructures. It is based on the principle of OCT, with spatial filtering characteristic of RCM incorporated through line illumination and detection. A LC-OCT prototype has been applied to the imaging of various human skin lesions, including carcinomas and melanomas in vivo to evaluate the potential of this technology in dermatology.

\section{Technical Limitations of Optical Coherence Tomography}

OCT can be classified into two main categories such as timedomain (TD)-OCT and frequency-domain (FD)-OCT. ${ }^{22}$ In TD-OCT, the sample reflectivity profile as a function of depth is acquired point by point by scanning the sample depth (A-scan). In FD-OCT, the sample reflectivity profile as a function of depth is acquired in parallel by analyzing the spectrum of the acquired interferometric signal. In both methods, a B-scan image is then obtained using lateral scanning of the light beam to acquire several adjacent A-scans.

FD-OCT has proven to be superior in terms of acquisition rate and detection sensitivity ${ }^{23}$ but presents shortcomings, notably a limited lateral resolution. ${ }^{22}$ Because all points along the depth range need to be in focus simultaneously, a depth of field (DOF) at least equal to the depth range is needed, which limits the beam focusing. Several approaches have been investigated to face the resulting intrinsic limitation of the lateral resolution in FD-OCT. One approach consists of extending the DOF by illuminating the sample with a Bessel beam $^{24-26}$ or by using appropriate phase masks. ${ }^{27}$ Alternative methods based on computational imaging solutions have also been proposed, including interferometric synthetic aperture microscopy ${ }^{28}$ and digital refocusing. ${ }^{29-31}$ Another approach consists of combining several B-scan images acquired at different depths over a reduced DOF with high lateral resolution. This approach has been implemented both by using the Gaborbased fusion method ${ }^{32}$ and by using multiple light beams focused at different depths. ${ }^{33}$ The latter approach, implemented in the Vivosight FD-OCT device commercialized by the company Michelson Diagnostics for skin imaging, yields a lateral resolution of $7 \mu \mathrm{m}$. $^{33}$

Unlike FD-OCT, TD-OCT offers the possibility of adjusting the focus continuously as a function of depth, making TD-OCT more attractive for the production of images with high lateral resolution. Dynamic focus tracking in TD-OCT with freespace optics has been reported, but the tracking rate was slow. ${ }^{34}$ A microelectromechanical systems mirror was designed for high-speed dynamic focus tracking, but without demonstration of the imaging capability in biological tissues. ${ }^{35}$ Another method consists of acquiring a sequence of images by gradually shifting the focus onto the sample and then fusing together the in-focus imaging zones. ${ }^{36} \mathrm{~A}$ trade-off between lateral resolution and image acquisition speed is then required. Yet another reported approach involves collecting multiple foci simultaneously with a multifocus fiber tip array. ${ }^{37}$ Despite these advances, however, TD-OCT imaging at high lateral resolution using dynamic focusing remains challenging as both a tracking speed on the order of a few $\mathrm{m} / \mathrm{s}$ and a repetition rate in the kilohertz range are needed.

An efficient approach for imaging with high lateral resolution is to acquire en face images rather than B-scans. In this configuration, there is no limitation to the beam focusing related to a DOF constraint. Optical coherence microscopy (OCM) is a version of OCT that acquires en face images using microscope objectives with a relatively high $\mathrm{NA}^{38}$ B-scan imaging with high lateral resolution can be achieved with OCM after acquiring a stack of en face images. ${ }^{39,40}$ Several OCM methods have been reported, depending on how the sample is illuminated. ${ }^{41}$ In the point-scanning method, an en face image is acquired by raster scanning a single spot of illumination, as with a confocal microscope. ${ }^{42}$ However, this method is not well suited for B-scan imaging in real time because of the acquisition time. OCM en face images can also be generated by only one-dimensional (1-D) scanning, using line illumination and detection. ${ }^{43}$ Although potentially faster, this method has not been adapted for high-speed B-scan imaging. At last, OCM en face images can be produced without scanning, through full-field illumination and parallel detection using an area camera. ${ }^{44,45}$ The full-field OCM (FF-OCM) method has the advantage of simplicity but suffers from higher incoherent scattered light. This is due to the absence of a confocal gate, which results in lower detection sensitivity when compared with point-scanning and line scanning OCM. ${ }^{41}$ A FF-OCM device (SkinTell) was commercialized by the company Agfa Healthcare for application in dermatology. B-scan imaging of skin, in vivo and in real time, is possible with the SkinTell device but with a significantly reduced penetration compared with conventional $\mathrm{OCT}^{46}$ and a reduced spatial resolution $(3 \mu \mathrm{m})$ compared with high-resolution FF-OCM. B-scan imaging of skin with high-resolution FF-OCM has also been demonstrated. ${ }^{47,48}$ However, since a stack of en face images (i.e., a three-dimensional dataset) had to be acquired to reconstruct sectional views in other orientations, B-scan imaging was limited in speed. Careful stabilization of the subject was required due to the long acquisition time.

\section{Principle of Line-Field Confocal Optical Coherence Tomography}

We have developed an OCT technique called LC-OCT to produce high-resolution B-scan images in real time with neither the lateral resolution limitation of FD-OCT nor the speed limitation 
of dynamically focused TD-OCT. The LC-OCT technique is based on a two-beam interference microscope with line illumination of the sample using a broadband light source and line detection using a line-scan camera. In LC-OCT, multiple A-scans are acquired in parallel. The depth scan can then be slower compared with conventional TD-OCT, without an increase in the acquisition time of a whole B-scan image. As the speed of the depth scan is significantly reduced, dynamic focusing is facilitated. A microscope objective can then be dynamically focused to image with high lateral resolution.

LC-OCT is basically a TD-OCT system with a parallel acquisition of multiple A-scans for B-scan imaging without lateral scanning of a light beam as required in conventional OCT. This parallelization facilitates dynamic focusing for B-scan imaging with high lateral resolution at a high (real-time) frame rate. LC-OCT can also be seen as a line-scanning OCM system, where the illumination line is scanned axially rather than laterally to acquire B-scan images.

An analogy of principle can be made between LC-OCT and FF-OCM by replacing the full-field illumination and area detection with line illumination and detection. The spatially incoherent light source used in FF-OCM is replaced in LC-OCT by a light source of high spatial coherence to generate a diffractionlimited line of light with considerably higher intensity.

A schematic diagram of the LC-OCT technique is shown in Fig. 1. It consists of a Michelson-type interferometer with a microscope objective placed in each arm. A supercontinuum laser is used as a source of spatially coherent broadband light. The collimated light beam passes through a cylindrical lens to achieve line illumination in the focal plane of the microscope objectives. The microscope objective in the interferometer reference arm is focused on the external side of a glass plate, thus constituting a low reflectivity $(\sim 4 \%)$ reference surface. An identical glass plate is placed in the sample arm in contact with the biological tissues to image. Immersion microscope objectives are employed with silicon oil as an immersion medium placed between the objectives and the glass plates.

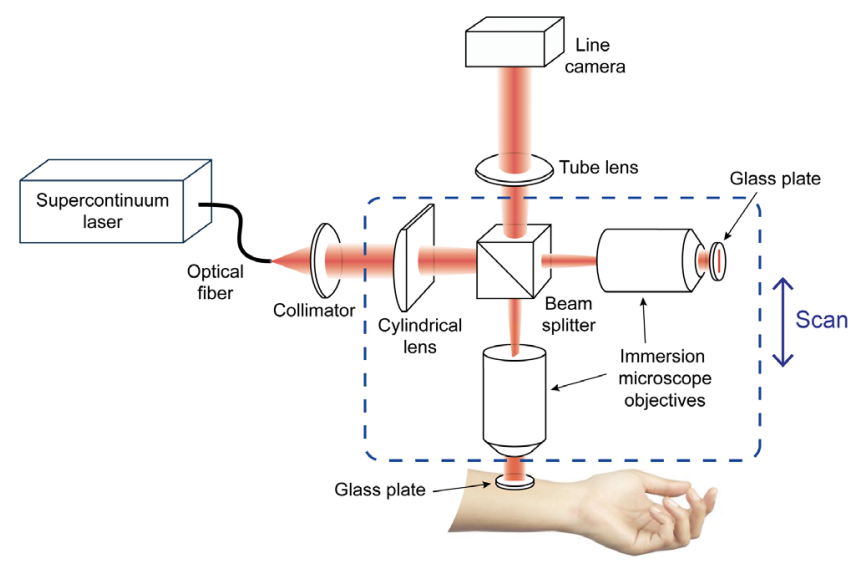

Fig. 1 Schematic of LC-OCT, combining the principles of OCT and line-scanning confocal microscopy. The prototype is based on a Linnik-type interference microscope, i.e., a Michelson interferometer with a microscope objective in each arm. The skin is illuminated by a line of broadband light generated by a supercontinuum laser. The interferometric signal is detected by a line-scan camera. The whole interferometer is displaced to scan the tissue in depth. A cross-sectional B-scan image of the backscattering skin microstructures is obtained in real-time by digital extraction of the interference fringe envelope.
This medium has an optical refractive index of about 1.4 in the near infrared, similar to the average refractive index of skin tissues. Despite the scan of the sample depth, optical dispersion mismatch in the interferometer arms is minimized to avoid degradation of the axial imaging resolution. ${ }^{49}$ This also ensures that the coherence plane (plane of zero optical path difference) and the focus always match ${ }^{49}$ and that the illumination line remains focused on these planes.

The imaging depth within the sample is scanned by displacement of the entire interferometer relative to the sample, as shown in Fig. 1, using a piezoelectric-driven linear stage. A phase-shifting algorithm is applied to extract the fringe envelope from the acquired interferometric data. The calculations are performed with a field-programmable gate array (FPGA) to optimize the operation speed. The result of these calculations is a flow of $\mathrm{B}$-scan images that are produced in real time. The images are shown in the logarithmic scale with autoadjusted contrast after being appropriately rescaled.

Line illumination and detection in LC-OCT, combined with the use of microscope objectives of relatively high numerical aperture (NA), provide a confocal gate, preventing most scattered light within the sample that does not contribute to the interferometric signal to be detected by the camera. ${ }^{43}$ Compared with conventional TD-OCT (with a large DOF), the lower detection dynamic range of a line scan camera than a photodiode is thus compensated (at least partially) by a more efficient confocal spatial filtering of unwanted light and an improved signal collection due to the use of an objective with higher NA. Compared with conventional FD-OCT, the more efficient confocal spatial filtering resulting from the use of an objective with higher NA enables LC-OCT to image in highly scattering tissues such as skin to a depth approaching that reached by FD-OCT. ${ }^{20}$ The confocal gate in LC-OCT, although less efficient than that achieved in point-scanning OCM, ${ }^{41}$ provides an advantage of LC-OCT over FF-OCM that has no confocal gate. The larger amount of incoherent scattered light with full-field illumination compared with line illumination reduces the maximum usable optical power before the camera saturates. The detection sensitivity and hence the image contrast and penetration depth are, therefore, improved with line illumination. ${ }^{41,43,45}$ Imaging in skin can be achieved with LC-OCT to a depth larger than that reached by FF-OCM. ${ }^{20,45,47}$

\section{Method}

\subsection{Line-Field Confocal Optical Coherence Tomography Setup}

A construction drawing of our LC-OCT prototype is shown in Fig. 2. The device is based on a Linnik interference microscope, i.e., a Michelon-type interferometer with a microscope objective placed in each arm. The microscope objective in one of the interferometer arms (reference arm) is focused on the external surface of a glass plate. An identical glass plate is placed in the other arm (sample arm) in contact with the biological tissues to image. The glass plates are made of fused silica and have a thickness of $500 \mu \mathrm{m}$. The light source is a supercontinuum laser (NKT photonics, SuperK Extreme). An average power of $50 \mathrm{~mW}$ is coupled into a single-mode fiber (Thorlabs, $780 \mathrm{HP}$ ) to the interferometer, which provides $25-\mathrm{mW}$ line illumination on the sample achieved with a plano-convex cylindrical lens of 58-mm-focal length (Thorlabs, LJ1918L1-B). Light from the sample and reference arms is recombined by a beam 


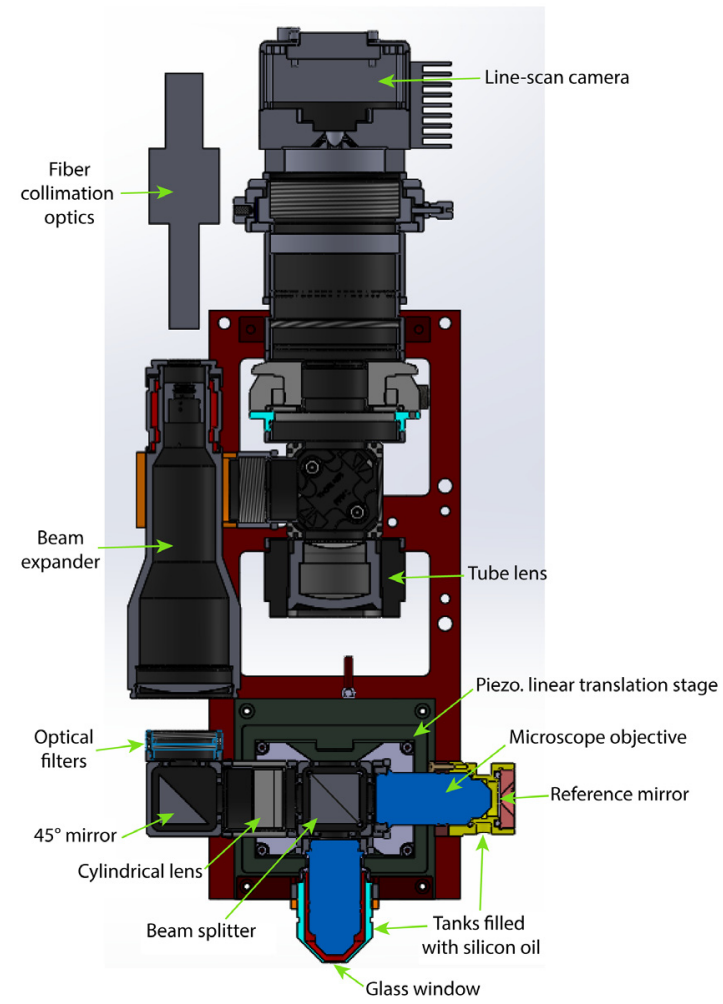

Fig. 2 Construction plan of the LC-OCT imaging head.

splitter (Thorlabs, BS017) and imaged, using a 200-mm-focal length tube lens (Thorlabs, ITL200) onto a line scan CCD camera (e2v AViiVA EM4). This camera has 2048 square pixels of size $14 \mu \mathrm{m}$, and the line acquisition rate is $70 \mathrm{kHz}$. The whole interferometer is mounted on a piezoelectric-driven linear stage (Physik Instrument, P628.1CD) for scanning the depth of the sample.

The device was designed for easy integration into healthcare institutions and research laboratories. It is in the form of a microscope whose inclination angle and height can be adjusted by the user to facilitate the examination of all skin regions directly on the patient (see Fig. 3). The head of the device ends with a glass window of $8-\mathrm{mm}$ diameter that is applied against the patient's

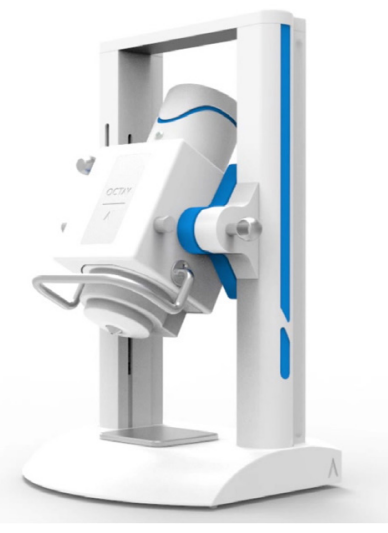

(a)

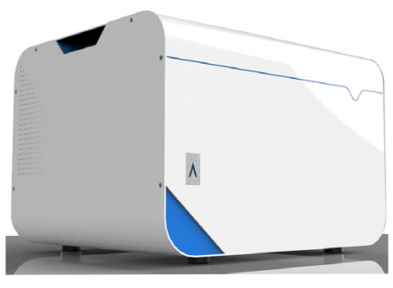

(b)
Fig. 3 LC-OCT device: (a) mobile imaging head and (b) central unit. skin. A central unit contains the supercontinuum laser, the piezoelectric servo controller, the computer, and the power supplies. The central unit is connected to the microscope through a wire bundle, protected with a sheath. The central unit provides several front-in ports: one universal serial bus (USB) port to connect USB devices, one Ethernet port to connect the device to an external network, and one high-definition multimedia interface (HDMI) port to connect the screen. A computer-embedded software has been developed for image acquisition and display on the screen, user interaction, and patient data management.

\subsection{Microscope Objectives and Immersion Medium}

Silicon oil is placed between the microscope objectives and the glass windows. Silicon oil is used as the immersion medium to mimic the optical refractive index and optical chromatic dispersion of skin tissues. This ensures that the coherence plane (plane of zero optical path difference) and the focus remain superimposed as the depth is scanned. ${ }^{49}$ Moreover, mismatch of the optical dispersion in the interferometer is avoided, or at least minimized. Microscope objectives of NA $=0.5$ and magnification $20 \times$ are chosen to provide sufficiently high lateral resolution along with a relatively large lateral FOV of $1.2 \mathrm{~mm}$. They have a working distance of $3.5 \mathrm{~mm}$, which allows for imaging through the $500-\mu \mathrm{m}$-thick glass windows plus down to about $1 \mathrm{~mm}$ below the tissue surface. Due to the unavailability of oil-immersion microscope objectives with these characteristics, water-immersion objectives are used (Olympus UMPLFLN 20XW).

\subsection{Image Acquisition, Processing and Display}

A five-frame phase-shifting algorithm is applied to extract the fringe envelope from the acquired interferometric data. This algorithm requires the arithmetic combination of five consecutive lines, $E_{1}, E_{2}, E_{3}, E_{4}, E_{5}$, according to $\left(E_{4}-E_{2}\right)^{2}-$ $\left(E_{1}-E_{3}\right)\left(E_{3}-E_{5}\right)$, with a phase difference between two consecutive lines of $\pi / 2 .^{50}$ The displacement of the piezoelectricdriven stage between the acquisition of two consecutive lines acquired, but the camera is adjusted to $\delta z=\bar{\lambda} /(8 n)$, where $\bar{\lambda}=$ $800 \mathrm{~nm}$ denotes the center optical wavelength of the detected light and $n=1.4$ the refractive index of the immersion medium (equal to the average refractive index of skin). This displacement generates an optical phase-shift of $\sim \pi / 2$ in the interferometer. The number of lines acquired during the scan of the depth over a range of $\Delta z=500 \mu \mathrm{m}$ is $N=\Delta z / \delta z \approx 7000$. A stacks of $\sim 7000$ lines are acquired continuously as the piezoelectricdriven stage oscillates in a sawtooth-like manner.

The calculation of the intensity-based B-scan images is performed in real-time at $10 \mathrm{frame} / \mathrm{s}$ using an FPGA. The images are shown in the logarithmic scale for dynamic compression with auto-adjusted contrast after being appropriately rescaled. Each image has a size of $2048 \times 875$ pixels (lateral $\times$ vertical), encoded on 32 bits. Images are saved in a TIFF format. In addition, images can be recorded as a 10-second video, also displayable in the TIFF format.

\subsection{Imaging Protocol}

The evaluation of the LC-OCT device was performed at SaintEtienne hospital under an institutional review board-approved protocol (Ref. RCB: 2016-A00319-42). Patients with suspicious skin lesions were identified by their physicians and 


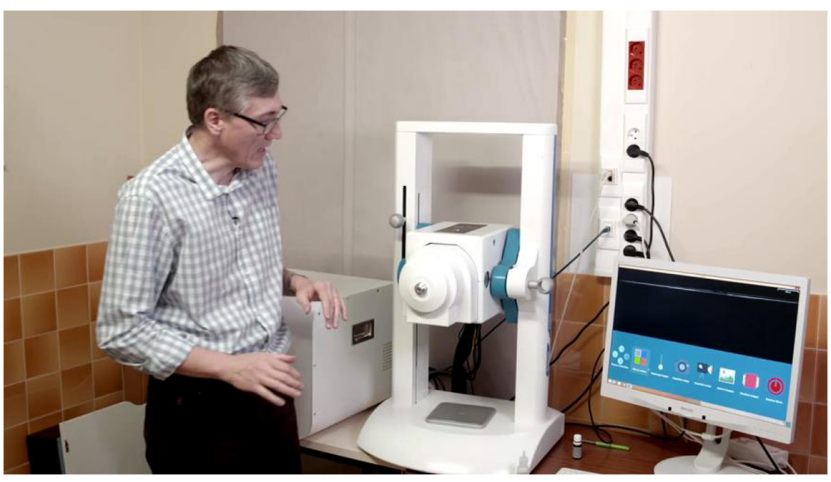

Fig. 4 Demonstration of use of our LC-OCT prototype by Pr. JeanLuc Perrot (MD) (Video 1, 27,497 KB [URL: https://doi.org/10.1117/1 .JBO.23.10.106007.1]).

offered an opportunity to participate in this study. Following informed and written consents, the suspicious area was clinically (visually) assessed using surface epiluminescent microscopy (dermoscopy), as per routine practice. The sterile glass window serves as a mechanical interface between the objective lens of the probe and the skin. After adding a drop of paraffin oil between the skin and the glass window, this window is applied to the surface of the patients' skin, gently flattening and stabilizing the area to be imaged. The purpose of the paraffin oil is to provide refractive index matching between the window and the skin, diminishing the specular back-reflection from both the skin surface and the glass window. Imaging was performed with LC-OCT at 10 frames/s (see Fig. 4). A series of about 20 images were recorded for each lesion. After LCOCT imaging, the lesions were surgically removed according to the routine practice protocols. The excised tissues were then processed for histopathological examination. Following chemical fixation, dehydration, and clearing, the tissues were embedded in paraffin wax and sectioned in slices using a microtome. The histological sections were then stained using the common haemotoxylin and eosin (H\&E) staining system for observation using conventional optical microscopy. Microscope objectives with $20 \times$ magnification were employed. This is the same magnification provided by the objectives used in the LC-OCT device.

\section{Line-Field Confocal Optical Coherence Tomography Performance}

\subsection{Axial Resolution}

The axial resolution of the LC-OCT images is determined theoretically by both the temporal coherence of the detected light (similar to OCT) and the DOF of the microscope objectives (similar to confocal microscopy). ${ }^{38}$ With a supercontinuum laser as a light source, the spectrum of detected light corresponds to the whole spectral response of the camera, centered at the wavelength of $\sim 800 \mathrm{~nm}$ with a full-width at half maximum (FWHM) of $\sim 250 \mathrm{~nm}$. The corresponding temporal coherence length, being significantly smaller than the DOF of the 0.5-NA microscope objectives, essentially determines the axial resolution. The axial response of the LC-OCT system was measured by imaging the interface between a glass plate and air in the sample arm, without using a sample. The FWHM of the imaged interface was $1.15 \mu \mathrm{m} \pm 0.01 \mu \mathrm{m}$, which is considered to be the axial imaging resolution. The theoretical resolution assuming a Gaussian-shaped spectrum centered at $800 \mathrm{~nm}$ with an FWHM of $250 \mathrm{~nm}$ is $0.80 \mu \mathrm{m}^{22}$ The difference between theory and experiment is attributed to residual dispersion mismatch in the interferometer and to the influence of the spectral transmittance of the optical components. The measured axial resolution is about five times better than the one offered by the Vivosight OCT device and three times better than the one offered by the SkinTell FF-OCM device. $^{20,33}$

\subsection{Lateral Resolution}

The NA and magnification of the microscope objectives used in LC-OCT can be chosen depending on the desired lateral resolution and FOV. Using microscope objectives of NA $=0.5$, a lateral resolution of $1.3 \mu \mathrm{m} \pm 0.1 \mu \mathrm{m}$ was measured from the FWHM of the illumination line in the microscope objective focal plane, in the direction perpendicular to the line. This surpasses the resolution of all currently available OCT devices and is similar to the confocal microscope commercialized for skin imaging (Vivascope, Caliber ID). ${ }^{20}$ Microscope objectives with NA values even $>0.5$ could be used, albeit with a reduction of the lateral FOV. The NA value of 0.5 was chosen so that the

Table 1 Features of state-of-the-art optical technologies commercialized for in vivo skin imaging.

\begin{tabular}{|c|c|c|c|c|c|}
\hline Technology & View & $\begin{array}{l}\text { Axial } \\
\text { resolution } \\
(\mu \mathrm{m})\end{array}$ & $\begin{array}{l}\text { Lateral } \\
\text { resolution } \\
(\mu \mathrm{m})\end{array}$ & $\begin{array}{l}\text { Lateral } \\
\text { field } \\
(\mathrm{mm})\end{array}$ & $\begin{array}{l}\text { Penetration } \\
\quad(\mathrm{mm})\end{array}$ \\
\hline $\begin{array}{l}\text { Reflectance Confocal Microscopy- } \\
\text { Vivascope }^{\circledR}\end{array}$ & En face & 5 & 1 & $1.0^{\mathrm{a}}$ & 0.2 \\
\hline OCT (multibeam FD-OCT)—Vivosight ${ }^{\circledR}$ & En face, B-scan, 3-D & 5 & 7.5 & 6 & 1 \\
\hline OCM (FF-OCM) $)^{\mathrm{b}}$-SkinTell ${ }^{\circledR}$ & En face, B-scan, 3-D & 3 & 3 & 1.5 & 0.3 \\
\hline Nonlinear microscopy-Dermalnspect ${ }^{\circledR}$ & En face & 2 & 1 & 0.35 & 0.2 \\
\hline LC-OCT ${ }^{\mathrm{C}}$ & B-scan & 1 & 1 & 1.2 & 0.5 \\
\hline
\end{tabular}

${ }^{a} \mathrm{An}$ area of $8 \times 8 \mathrm{~mm}$ can be acquired through montage image.

${ }^{\mathrm{b}}$ The SkinTell ${ }^{\circledR}$ (Agfa Healthcare) device is no longer commercialized.

${ }^{\mathrm{C}} \mathrm{A}$ LC-OCT device is planned to be marketed by DAMAE Medical at the end of 2019. 
axial and lateral resolutions are close, which is desirable for B-scan imaging. The isotropic spatial resolution of $\sim 1.0 \mu \mathrm{m}$ achieved with LC-OCT is close to the resolution of conventional histological images. A comparison of spatial resolution achieved with current optical technologies for skin imaging is shown in Table 1.

\subsection{Detection Sensitivity and Acquisition Time}

The detection sensitivity, defined as the smallest detectable reflectivity, was considered to be the mean value of the background noise in the images. It can be expressed as an equivalent reflectivity coefficient by comparison with the signal measured from an interface with a known reflectivity. Using the plane interface between the glass window and the immersion oil as a calibration sample of reflectivity equal to $7.7 \times 10^{-5}$, the detection sensitivity was evaluated at $-86 \mathrm{~dB}$, which is close to the theoretical value of $-88 \mathrm{~dB}$ assuming a shot-noise limited detection. $^{45}$

A B-scan is obtained from a stack of $\sim 7000$ line interferometric images acquired by the line scan camera, which represents a scan of the depth within the tissues over a distance of $\sim 500 \mu \mathrm{m}$ (image axial field). Each line of the B-scan images is calculated from the algebraic combination of five successive line interferometric images. The time required to acquire each line of the B-scan images is, therefore, equal to $5 / f_{\text {camera }}=71 \mu \mathrm{s}$, where $f_{\text {camera }}=70 \mathrm{kHz}$ is the frame rate of the camera. This acquisition time of a few tens of microseconds is similar to the acquisition time of each column of a $\mathrm{B}$-scan image in conventional FD-OCT. ${ }^{22}$ It is short enough to avoid blurring of the phase-sensitive interferometric signal that may occur due to sample motion. In LC-OCT, as in FD-OCT, motion may however have an impact resulting in a geometrical distortion of the image. ${ }^{51}$ This artifact is avoided by the mechanical stabilization achieved by pressing the skin against the glass plate (see Fig. 1) maintained at a fixed position under the microscope objective. The LC-OCT B-scan images are shown at 10 frame/s, which allows for real-time examination of skin tissues.

\section{Skin Imaging with Line-Field Confocal Optical Coherence Tomography}

\subsection{Healthy Skin Imaging}

The performance of LC-OCT for skin imaging has first been evaluated by imaging healthy skin. Figure 5(a) shows an example image of healthy skin (phototype 2) obtained from a 25-yearold man on the back of the hand. The epidermis and the dermis can be clearly distinguished. They are separated by the dermalepidermal junction (DEJ). Several layers can be identified in the epidermis: the stratum corneum (SC), the stratum granulosum (SG), and the stratum spinosum (SS). The nuclei of keratinocytes $(\mathrm{KN})$ in the epidermis are resolved and appear as small, round, black structures in the image. The dermis is composed mainly of collagen fibers (CF) and blood vessels (BV). These biological structures can be distinguished in the images. Figure 5(b) shows an example LC-OCT image of healthy skin (phototype 5) obtained from a 23-year-old woman on the back of the hand. Here, the DEJ is much more reflective because of the higher amount of melanin in darker phototypes. Indeed, the stratum basale (SB) can be easily distinguished in LC-OCT images of dark-skinned individuals.

\subsection{Skin Cancer Imaging}

LC-OCT has been applied to the imaging of various human skin lesions, in vivo, including carcinomas and melanomas. A total of 105 lesions on 86 patients have been imaged at Saint-Etienne hospital.

An example LC-OCT image of basal cell carcinoma (BCC) is shown in Fig. 6. The corresponding Hematoxylin-Eosin (H\&E) stained histopathological image is shown for comparison. LC-OCT correlates well with histopathology, as it is able to show the features that make the diagnosis possible. These features include the presence of lobulated structures (tumor islands or nests) within the dermis, which are separated by the tumor stroma. A dark cleft between lobules and the adjacent tumor stroma is visible, likely due to mucin deposition. A bright rim that correlates with characteristic high-density collagen surrounds the lobules. Moreover, it is possible to assess the
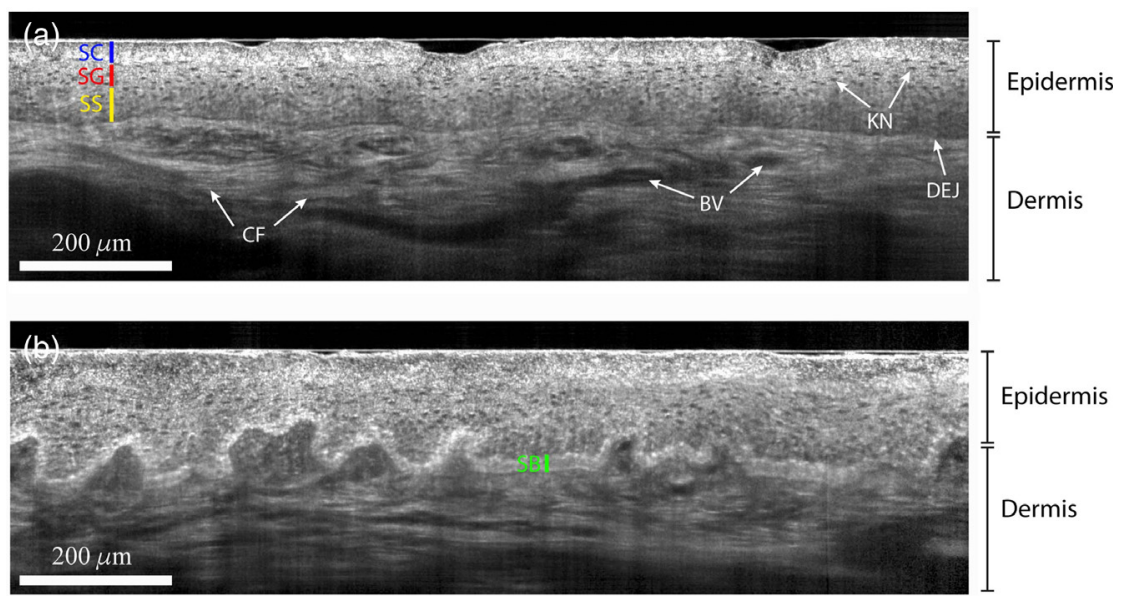

Fig. 5 LC-OCT images of healthy human skin on the back of the hand from (a) a 25-year-old man, phototype 2 and (b) a 23-year-old woman, phototype 5. SC, stratum corneum layer; SG, stratum granulosum layer with stretch nuclei; SS, stratum spinosum layer with roundish nuclei; SB, stratum basale rich in melanin in darker phototypes; CF, collagen fibers; BV, blood vessel; KN, nuclei of keratinocytes; DEJ, dermal-epidermal junction. 


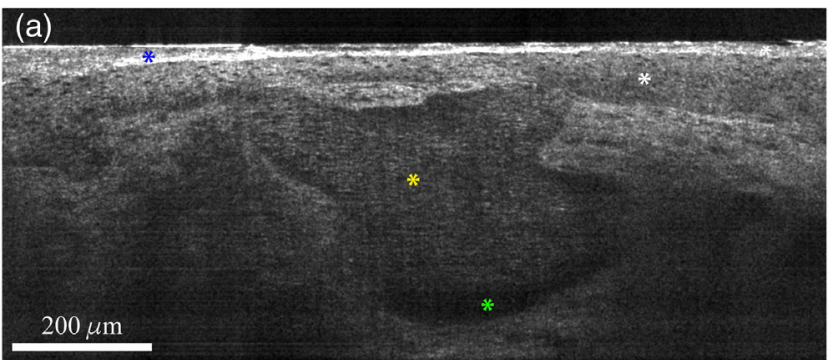

(b)

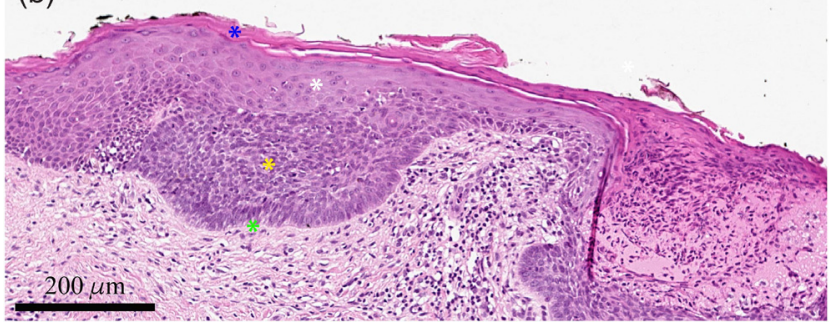

Fig. 6 (a) LC-OCT image of a superficial BCC and (b) corresponding histopathological examination with Hematoxylin-Eosin stain. Proliferating nests of tumor cells (tumor islands) are connected to the epidermis and extend into the superficial dermis. Blue star: SC; white star: epidermis; yellow star: clusters of tumor cells that are poorly reflecting in the LC-OCT image; green star: cleft between the tumor islands and the dermis.

shape and the location of the lobules, which are crucial to ascertain the BCC subtype. As is shown in this case of superficial $\mathrm{BCC}$, for example, the tumor islands are connected to the epidermis.

An example image of melanoma is shown in Fig. 7. The LCOCT image correlates well with histopathology. LC-OCT is able to show most of the key histopathological features that are used for melanoma diagnosis: general architectural disarrangement, consumption of the epidermis, partial disruption of the dermal-epidermal junction, presence of dermal nests of atypical
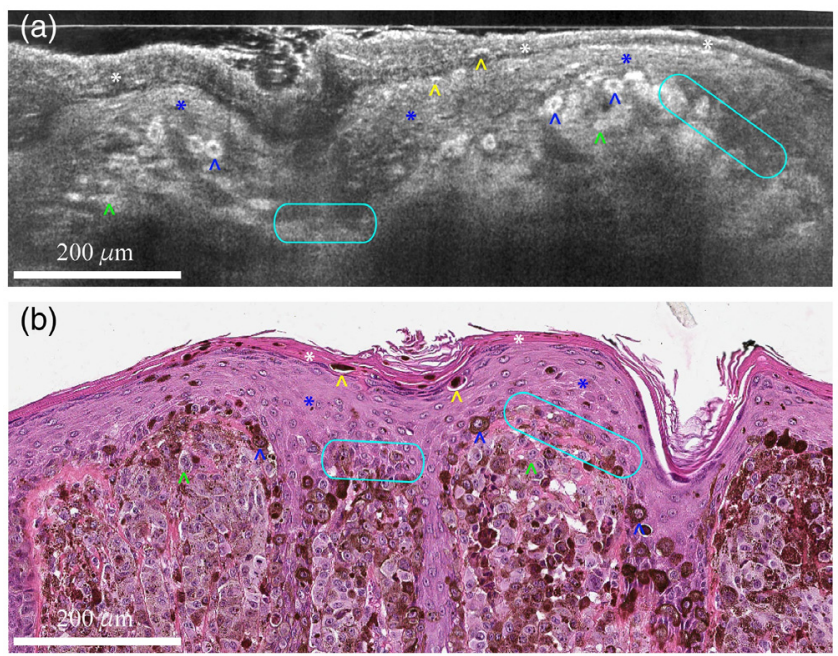

Fig. 7 (a) LC-OCT image of human skin melanoma and (b) corresponding H\&E histopathology image. White star: SC; blue star: epidermis; blue arrowhead: pagetoid spread (epidermal invasion) of epithelial tumor cells; yellow arrowhead: intracorneal tumor cells in the process of being eliminated; green arrowhead: dermal clusters of melanocytic tumor cells; turquoise circles: partial disruption of the dermal-epidermal junction.
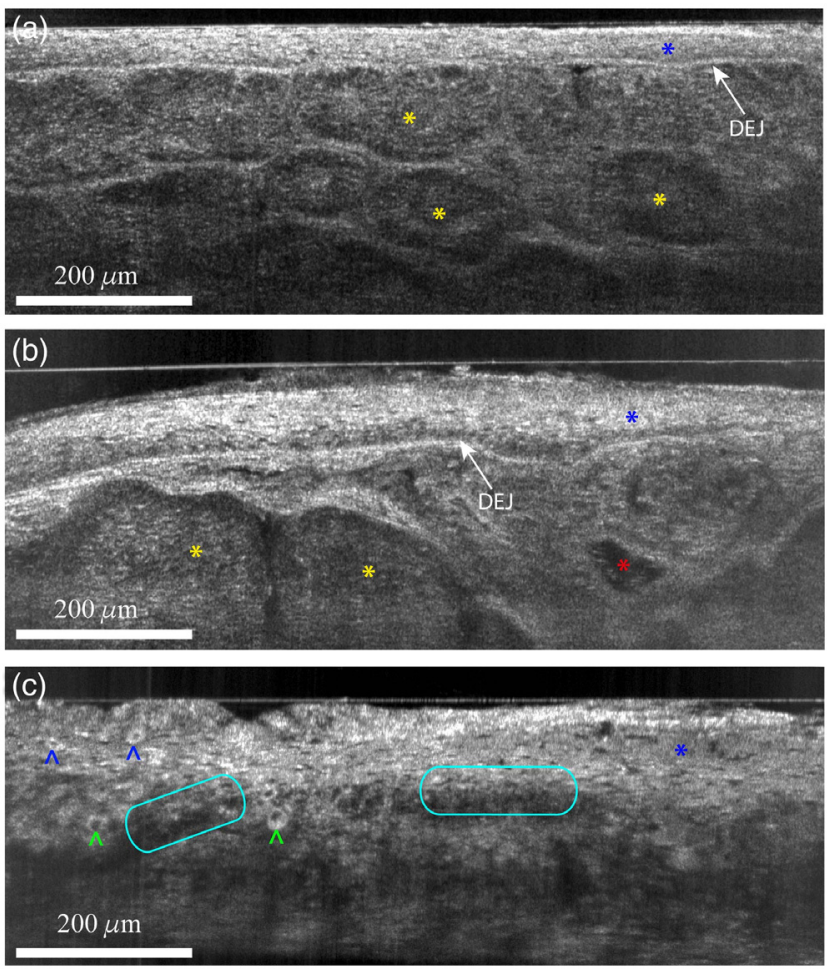

Fig. 8 LC-OCT images of human skin lesions. (a) Nodular BCC. Blue star: epidermis; yellow star: tumor islands separated from the epidermis. Note the typical dark cleft and bright rim (stroma reaction) surrounding each lobule. DEJ: dermal-epidermal junction. (b) Nodular BCC. Blue star: epidermis; yellow star: tumor islands separated from the epidermis. Red star: dilated vessel. (c) Melanoma. Blue star: epidermis; blue arrowhead: pagetoid spread (epidermal invasion) of melanocytic tumor cells; green arrowhead: dermal clusters of melanocytic tumor cells; turquoise circles: partial disruption of the dermal-epidermal junction.

melanocytes of various size and shape, and pagetoid spread of atypical melanocytes within the epidermis. Other examples of skin lesions imaged with LC-OCT, including nodular BCCs and a melanoma, are shown in Fig. 8.

\section{Discussion and Conclusion}

The use of noninvasive imaging techniques in dermatology has been reported to improve the diagnostic accuracy and the practice of biopsies and at the same time to reduce the need for tissue excision. However, the current clinically available imaging techniques do not yet entirely meet the need for early and accurate, noninvasive detection of skin cancers.

RCM provides high imaging resolution but is limited by a relatively weak skin penetration and the en face orientation of the images, which hampers the diagnosis of certain nonmelanoma skin cancers. Similarly, nonlinear optical microscopy provides high resolution but has the limitations of relatively weak penetration, en face orientation of the images and high costs. Conversely, OCT provides vertically oriented images with deeper skin penetration but is limited by its insufficient imaging resolution, which renders the diagnosis of conditions such as melanoma more challenging.

As a response to these issues, we have developed LC-OCT, an imaging technique that combines the advantages of the currently available optical techniques in terms of spatial resolution, penetration, and image orientation. LC-OCT delivers vertically 
oriented sectional images of skin in real time (10 frames/s) with a quasi-isotropic spatial resolution of $\sim 1 \mu \mathrm{m}$, revealing a comprehensive morphological mapping of tissues at a cellular level, down to a depth of $\sim 500 \mu \mathrm{m}$, over a lateral FOV of $1.2 \mathrm{~mm}$.

LC-OCT is basically a parallelized TD-OCT system using line illumination and detection for B-scan imaging without lateral scanning of a light beam as required in conventional OCT. Due to the parallelization of the acquisition, the speed of the depth scan can be reduced, which facilitates continuous dynamic focusing. A microscope objective is dynamically focused to produce B-scan images in real time with high lateral resolution. Moreover, line illumination and detection, combined with the use of a relatively high NA objective, provide an efficient confocal gate, which prevents most of unwanted scattered light from being detected. Using a supercontinuum laser as a light source and balancing the optical dispersion in the interferometer arms, the axial resolution in LC-OCT reaches the best axial resolution achieved in OCT at a comparable central optical wavelength.

Our LC-OCT prototype has been applied to the in vivo imaging of various skin lesions, including carcinomas and melanomas. Surgical excisions of the lesions have then been performed followed by tissue processing to realize H\&Estained histopathological images. The spatial resolution, orientation, and imaging contrast mechanism of the LC-OCT images allowed for a high level of similarity with the conventional histopathological images. LC-OCT was able to show most of the histopathological elements that allow for medical diagnosis.

Following the pilot study carried out at St-Etienne hospital, a multicenter clinical trial is due to start at the end of 2018 with the goal of further evaluating the clinical interest of LC-OCT in dermatology. A handheld prototype of the LC-OCT technology is currently under development. This handheld probe, with a smaller imaging head, will facilitate the use of LCOCT by dermatologists in daily practice and enable the imaging of difficult-to-access regions of the skin. The current disadvantage of a lateral FOV limited to $1.2 \mathrm{~mm}$ may then be compensated by the possibility of easily scanning the probe on the skin surface. Using such a tool could help improve clinical diagnostic accuracy, allowing for the early detection of malignant skin tumors-including melanoma-and a reduction in the number of surgical excisions of benign lesions. Other promising areas of application for LC-OCT include the imaging of lesions, where conventional surgical excisions would be hazardous or impossible and the guidance of surgical interventions through identification of tumor margins. In addition, LC-OCT may also be used to identify the optimal area to biopsy in the case of extended lesions, thus reducing false negative rates from sampling errors.

\section{Disclosures}

The authors thank the "Ministère de l'Enseignement Supérieur et de la Recherche (MESR)" and the "Banque Publique d'Investissement France (BPI France)" for financial supports.

\section{Acknowledgments}

The authors are grateful to Cyril Habougit, medical doctor at Saint-Etienne hospital for the histology preparations and obtaining the histopathological images. They are also grateful to Frederique Vanholsbeeck, professor of Optical Physics at the university of Auckland, for fruitful discussions. They thank Katie Fletcher for contributing to the manuscript edition.

\section{References}

1. Z. Apalla et al., "Epidemiological trends in skin cancer," Dermatol. Pract. Concept 7, 1-6 (2017).

2. M. Mc Carthy, "US melanoma prevalence has doubled over past 30 years," Br. Med. J. 350, h3074 (2015).

3. A. M. Glazer et al., "Clinical diagnosis of skin cancer: enhancing inspection and early recognition," Dermatol. Clin. 35, 409-416 (2017).

4. N. Woltsche et al., "Dermoscopy in the era of dermato-oncology: from bed to bench side and retour," Expert Rev. Anticancer Ther. 16, 531541 (2016).

5. S. R. Fuller et al., "Digital dermoscopic monitoring of atypical nevi in patients at risk for melanoma," Dermatol. Surg. 33, 1198-1206 (2007).

6. H. Kittler et al., "Diagnostic accuracy of dermoscopy," Lancet Oncol. 3, 159-165 (2002).

7. N. Kollias and G. N. Stamatas, "Optical non-invasive approaches to diagnosis of skin diseases," J. Investig. Dermatol. Symp. Proc. 7, 64-75 (2002).

8. C. Fink and H. A. Haenssle, "Non-invasive tools for the diagnosis of cutaneous melanoma," Skin Res. Technol. 23, 261-271 (2017).

9. K. Konig and I. Riemann, "High-resolution multiphoton tomography of human skin with subcellular spatial resolution and picosecond time resolution," J. Biomed. Opt. 8, 432-439 (2003).

10. G. Pellacani et al., "The impact of in vivo reflectance confocal microscopy for the diagnostic accuracy of melanoma and equivocal melanocytic lesions," J. Invest. Dermatol. 127, 2759-2765 (2007).

11. P. Guitera et al., "The impact of in vivo reflectance confocal microscopy on the diagnostic accuracy of lentigo maligna and equivocal pigmented and nonpigmented macules of the face," J. Invest. Dermatol. 130, 20802091 (2010).

12. I. Alarcon et al., "Impact of in vivo reflectance confocal microscopy on the number needed to treat melanoma in doubtful lesions," $\mathrm{Br} . J$. Dermatol. 170, 802-808 (2014).

13. J. Champin et al., "In vivo reflectance confocal microscopy to optimize the spaghetti technique for defining surgical margins of lentigo maligna," Dermatol. Surg. 40, 247-256 (2014).

14. D. Huang et al., "Optical coherence tomography," Science 254, 11781181 (1991).

15. J. Welzel, "Optical coherence tomography of the human skin," J. Am. Acad. Dermatol. 37, 958-963 (1997).

16. J. Welzel, "Optical coherence tomography in dermatology: a review," Skin Res. Technol. 7, 1-9 (2001).

17. M. A. Boone et al., "Imaging of basal cell carcinoma by high-definition optical coherence tomography: histomorphological correlation. A pilot study," Br. J. Dermatol. 167, 856-864 (2012).

18. A. J. Coleman et al., "Histological correlates of optical coherence tomography in non-melanoma skin cancer," Skin Res. Technol. 19, e10-e19 (2013).

19. M. Ulrich et al., "The sensitivity and specificity of optical coherence tomography for the assisted diagnosis of nonpigmented basal cell carcinoma: an observational study," Br. J. Dermatol. 173, 428-435 (2015).

20. A. Levine, K. Wang, and O. Markowitz, "Optical coherence tomography in the diagnosis of skin cancer," Dermatol. Clinics 35, 465-488 (2017).

21. W. R. Zipfel, R. M. Williams, and W. W. Webb, "Nonlinear magic: multiphoton microscopy in the biosciences," Nat. Biotechnol. 21, 13691377 (2003).

22. A. G. Podoleanu, "Optical coherence tomography," J. Microsc. 247, 209-219 (2012).

23. M. A. Choma et al., "Sensitivity advantage of swept source and Fourier domain optical coherence tomography," Opt. Express 11, 2183-2189 (2003).

24. Z. Ding et al., "High-resolution optical coherence tomography over a large depth range with an axicon lens," Opt. Lett. 27, 243-245 (2002).

25. R. A. Leitgeb et al., "Extended focus depth for Fourier domain optical coherence microscopy," Opt. Lett. 31, 2450-2452 (2006).

26. K. S. Lee and J. P. Rolland, "Bessel beam spectral-domain high-resolution optical coherence tomography with micro-optic axicon providing extended focusing range," Opt. Lett. 33, 1696-1698 (2008). 
27. J. Mo, M. de Groot, and J. F. de Boer, "Focus-extension by depthencoded synthetic aperture in optical coherence tomography," Opt. Express 21, 10048-10061 (2013).

28. T. S. Ralston et al., "Interferometric synthetic aperture microscopy," Nat. Phys. 3, 129-134 (2007).

29. L. Yu et al., "Improved lateral resolution in optical coherence tomography by digital focusing using two- dimensional numerical diffraction method," Opt. Express 15, 7634-7641 (2007).

30. G. Liu, Z. Zhi, and R. K. Wang, "Digital focusing of OCT images based on scalar diffraction theory and information entropy," Biomed. Opt. Express 3, 2774-2783 (2012).

31. A. Grebenyuk et al., "Numerically focused full-field swept-source optical coherence microscopy with low spatial coherence illumination," Appl. Opt. 53, 1697-1708 (2014).

32. J. P. Rolland et al., "Gabor-based fusion technique for optical coherence microscopy," Opt. Express 18, 3632-3642 (2010).

33. J. Holmes and S. Hattersley, "Image blending and speckle noise reduction in multi-beam OCT," Proc. SPIE 7168, 71681N (2009).

34. J. M. Schmitt, S. L. Lee, and K. M. Yung, "An optical coherence microscope with enhanced resolving power in thick tissue," Opt. Cоттип. 142, 203-207 (1997).

35. B. Qi et al., "Dynamic focus control in high-speed optical coherence tomography based on a microelectromechanical mirror," Opt. Commun. 234, 443-448 (2004).

36. W. Drexler et al., "In vivo ultrahigh-resolution optical coherence tomography," Opt. Lett. 24, 1221-1223 (1999).

37. V. X. D. Yang et al., "Micromachined array tip for multifocus fiber-based optical coherence tomography," Opt. Lett. 29, 1754-1756 (2004).

38. J. A. Izatt et al., "Optical coherence microscopy in scattering media," Opt. Lett. 19, 590-592 (1994)

39. H. C. Lee et al., "Ultrahigh speed spectral-domain optical coherence microscopy," Biomed. Opt. Express 4, 1236-1254 (2013).

40. V. J. Srinivasan et al., "Optical coherence microscopy for deep tissue imaging of the cerebral cortex with intrinsic contrast," Opt. Express 20, 2220-2239 (2012).
41. Y. Chen et al., "Improved detection sensitivity of line-scanning optical coherence microscopy," IEEE J Sel. Top. Quantum Electron. 18, 10941099 (2012).

42. A. D. Aguirre et al., "High-resolution optical coherence microscopy for high-speed, in vivo cellular imaging," Opt. Lett. 28, 2064-2066 (2003).

43. Y. Chen et al., "High-resolution line-scanning optical coherence microscopy," Opt. Lett. 32, 1971-1973 (2007).

44. E. Beaurepaire et al., "Full-field optical coherence microscopy," Opt. Lett. 23, 244-246 (1998).

45. A. Dubois et al., "Ultrahigh-resolution full-field optical coherence tomography," Appl. Opt. 43, 2874-2882 (2004).

46. S. Schuh et al., "Comparison of different optical coherence tomography devices for diagnosis of non-melanoma skin cancer," Skin Res. Technol. 22, 395-405 (2016).

47. E. Dalimier and D. Salomon, "Full-field optical coherence tomography: a new technology for 3D high-resolution skin imaging," Dermatology 224, 84-92 (2012).

48. J. Ogien and A. Dubois, "A compact high-speed full-field optical coherence microscope for high resolution in vivo imaging," J. Biophotonics (2018).

49. A. Dubois, "Focus defect and dispersion mismatch in full-field optical coherence microscopy," Appl. Opt. 56, D142-D150 (2017).

50. K. G. Larkin, "Efficient nonlinear algorithm for envelope detection in white light interferometry," J. Opt. Soc. Am. A 13, 832-843 (1996).

51. S. H. Yun et al., "Motion artifacts in optical coherence tomography with frequency-domain ranging," Opt. Express 12, 2977-2998 (2004).

Arnaud Dubois received his $\mathrm{PhD}$ in physics from Paris-Sud University in 1997. Since 2006, he is a professor of optics at Institut d'Optique Graduate School, France. He has published 125 research articles in scientific journals and conference proceedings and 12 book chapters. He was the scientific editor in 2016 of the first and only handbook on full-field OCT. He has five patents to his credit. In 2014, he cofounded DAMAE Medical, a startup company working on an innovative OCT technique for skin imaging.

Biographies for the other authors are not available. 